\title{
Monte Carlo simulation of the effects of vacuum-ultraviolet radiation on dielectric materials
}

\author{
G. S. Upadhyaya, J. L. Shohet, ${ }^{\text {a) }}$ and J. L. Lauer \\ Plasma Processing and Technology Laboratory and Department of Electrical and Computer Engineering, \\ University of Wisconsin-Madison, Madison, Wisconsin 53706
}

(Received 4 October 2004; accepted 17 January 2005; published online 1 March 2005)

\begin{abstract}
Radiation-induced damage during plasma processing of semiconductor materials can adversely affect device reliability. However, it has been shown that vacuum ultraviolet (VUV) radiation (8-20 $\mathrm{eV}$ ) can beneficially deplete previously deposited charge on the surface of dielectrics by temporarily increasing their conductivity. Incident VUV photons can cause photoemission and form electron-hole pairs in the dielectric thus producing the desired increased conductivity. To verify this, statistical information obtained from a Monte Carlo simulation is used to model VUV exposure of dielectrics. The simulation calculates the surface potential on the dielectric produced by electron photoemission, which compares favorably with experimental surface-potential measurements made using a Kelvin probe. (C) 2005 American Institute of Physics. [DOI: 10.1063/1.1879100]
\end{abstract}

Charge deposition on dielectrics during plasma processing adversely affects device reliability. ${ }^{1}$ However, vacuumultraviolet (VUV) radiation emitted by the plasma can be beneficial and can deplete charge by temporarily increasing the conductivity of the dielectric. ${ }^{2-4}$ The increase is caused by electron-hole-pair production ${ }^{4}$ which occurs when photons of energy higher than the band-gap energy strike the dielectric. ${ }^{5}$

Electrons photoemitted from the dielectric lead to the formation of a positive potential. ${ }^{4}$ Electrons are emitted if they have sufficient energy to overcome this potential. Experimentally, the photoemission current first increases and then decreases as the potential on the substrate rises to a constant value as shown in Fig. 1. ${ }^{5}$

We hypothesize that a Monte Carlo ${ }^{6}$ simulation of VUV radiation interacting with a dielectric can predict the photoemitted dielectric surface-charge density and the resulting surface potential. To compare the simulation with experiment, the surface potential on a dielectric charged with a beam of VUV photons produced by a synchrotron was measured using a Kelvin probe. ${ }^{3}$

The process can be broken down into several parts. They are: (1) Photoelectric absorption, which leads to electronhole pair production, (2) electron excitation, and/or (3) photon (Rayleigh) scattering. If an electron-hole pair is produced, following the trajectory of the electron determines whether it is emitted or remains trapped in the dielectric. The emitted electrons contribute to a net charge buildup.

For energies between 8-20 eV, the dominant photonatom interaction processes are Rayleigh scattering and photoelectric absorption. ${ }^{7}$ At low energies $(\$ 500 \mathrm{eV})$, photoelectric absorption significantly dominates Rayleigh (elastic) scattering. ${ }^{8}$ In addition, Rayleigh scattering does not contribute to any significant energy loss because the photon is deflected only by a very small distance.

After electrons are excited into the conduction band, their dynamics are governed primarily by elastic scattering, which is included in the simulation. Inelastic scattering of the electrons, although small, cannot be neglected because

${ }^{a)}$ Electronic mail: shohet@engr.wisc.edu this determines the energy loss of an electron along its trajectory. If inelastic scattering were not included, an overestimation of the positive-charge density in the dielectric would occur since more electrons would be predicted to leave the dielectric. To correct for this, inelastic scattering for electrons is included using the continuous slowing down approximation (CSDA). ${ }^{9}$

The cross sections for photoelectric absorption were obtained from the form factor, attenuation, and scattering tables published by the National Institute of Standards and Technology (NIST). ${ }^{10}$ Since the uncertainty ${ }^{10}$ in these quantities at VUV energies can be a factor of 2 or higher, we use an approximate formula ${ }^{10}$ for the cross sections. We determine them from the mass photoabsorption coefficient $\mu$ given in Ref. 10 by use of Eq. (1)

$$
\mu=\frac{N_{A}}{M W} \sum_{i} x_{i} \sigma_{a i},
$$

where $M W$ is the molecular weight of the dielectric containing $x_{i}$ atoms of atomic weight $A_{i}$, i.e., $M W=\sum_{i} x_{i} A_{i}$. The

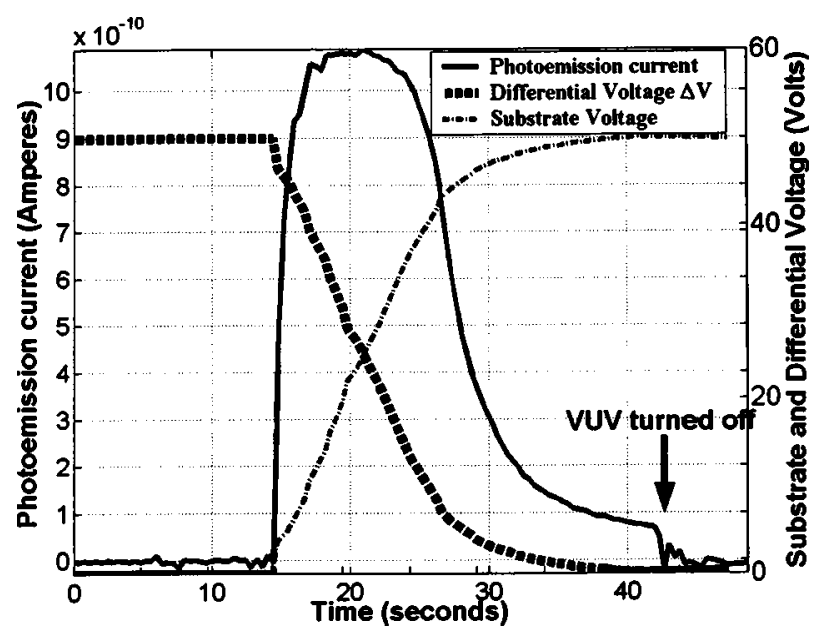

FIG. 1. (Color online) Experimentally measured photoemission current and substrate voltage during exposure of $\mathrm{Si}_{3} \mathrm{~N}_{4}$ to $20 \mathrm{eV}$ synchrotron radiation with a flux of $\sim 7 \times 10^{9}$ photons $/ \mathrm{s}$. 
weighted cross sections are $\sum_{i} x_{i} \sigma_{a i}$ and are used in the simulation.

When an electron-hole pair is formed, the electron energy is $E_{\text {photoelectron }}=E_{h \nu}-E_{\text {band gap }}$, where $E_{h \nu}(\mathrm{eV})$ is the photon energy and $E_{\text {band gap }}(\mathrm{eV})$ is the band-gap energy. Since we use a monoenergetic beam of photons, the electrons injected into the conduction band are also initially monoenergetic.

If an electron is emitted from the dielectric, it overcomes the potential (electron-affinity) barrier, which is of the order of $1-2 \mathrm{eV}$ for silicon nitride. The energy of a photoemitted electron is, therefore, $E_{\text {photoelectron }} \geqslant E_{h \nu}-E_{\text {band gap }}-E_{\text {aff }}$, where $E_{\text {aff }}(\mathrm{eV})$ is the energy required (electron affinity) for an electron to be removed from the conduction band and to be emitted from the dielectric. The photoelectrons in the above equation are not monoenergetic since their energy depends on the total distance that the electrons travel in the dielectric before being emitted.

To calculate the electron trajectory, we assume it undergoes a number of elastic and/or inelastic scatterings before it is either expelled or loses all of its energy. For elastic scattering, the trajectories are developed as follows. ${ }^{11}$ The distance $s$ traveled by an electron between successive scatterings is a random variable that follows the probability distribution given by

$$
p(s) d s=\frac{1}{\lambda} e^{-s / \lambda} d s,
$$

where $\lambda$ is the mean-free path given by

$$
\lambda=\frac{1}{n \sigma_{\mathrm{Mott}}},
$$

and $n$ is the density (atoms $/ \mathrm{cm}^{3}$ ), and $\sigma_{\text {Mott }}\left(\mathrm{cm}^{2}\right)$ is the Mott elastic scattering cross section. ${ }^{11} s$ is related to a random number $r$ which is uniform in the interval $[0,1]$ as $s=-\lambda \ln (r)$.

To determine the angular distribution of the scattered electrons, we use an empirical form for the differential elastic-scattering cross section. ${ }^{12}$ This cross section has two terms, the screened Rutherford cross section ${ }^{12}$ which accounts for elastic electron Coulomb scattering, and an isotropic scattering cross section. The latter is needed to correct for the fact that the Rutherford cross section is highly peaked in the forward-scattering direction and thus overestimates forward scattering. ${ }^{12}$

The ratio of the Rutherford to the isotropic cross sections is used to determine the scattering angle (polar angle) of the electrons. This is ${ }^{12}$

$$
\frac{\sigma_{\text {Rutherford }}}{\sigma_{\text {isotropic }}}=\frac{300 E^{(1-Z / 2000)}}{Z}+\frac{Z^{3}}{3 \times 10^{5} E},
$$

where $E(\mathrm{eV})$ is the photoelectron energy and $Z$ is the atomic number of the scattering center.

The probability of a given event to occur is proportional to the normalized magnitude of its corresponding cross section. ${ }^{13}$ Initially, a random number with uniform distribution between 0 and 1 determines if the scattering event is Rutherford or isotropic based on Eq. (4). For Rutherford scattering, the polar scattering angle in spherical coordinates is $\cos \theta=1-(2 \alpha m / 1+\alpha-m)$, where $m$ is another random number uniformly distributed between 0 and 1 , and $\alpha$ (dimensionless) is the Rutherford screening parameter, ${ }^{12}$ Downloaded 12 Feb 2007 to 128.104.198.69. Redistribution subje

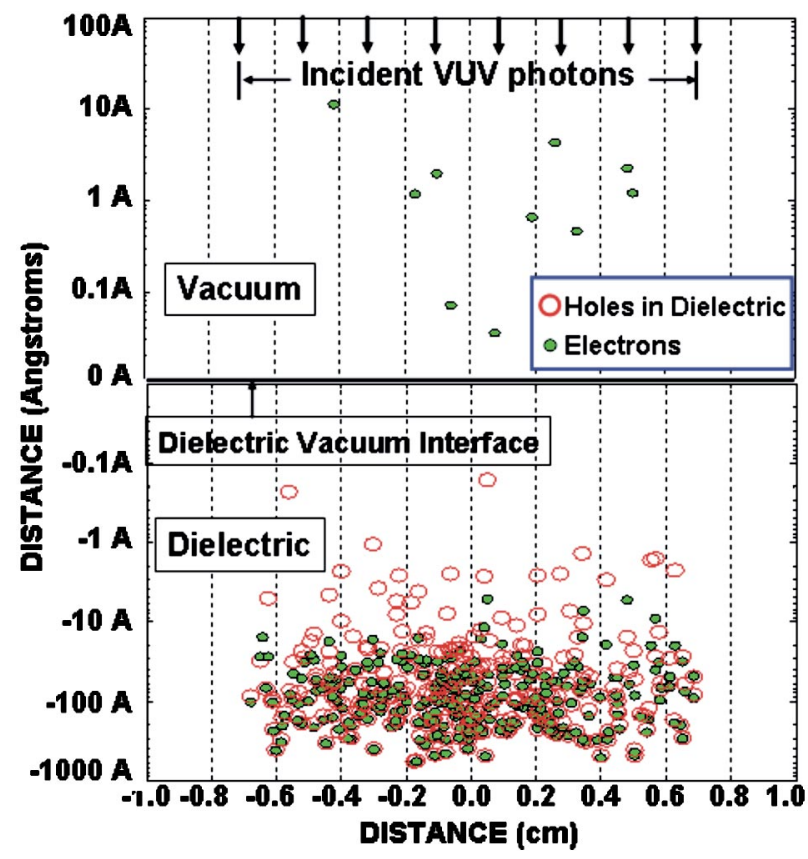

FIG. 2. (Color online) Location of emitted electrons and their companion holes remaining in the dielectric as calculated by the simulation during exposure to VUV radiation. Complete electron-hole pairs remaining in the dielectric are not shown.

$7 \times 10^{-3} / E$, where $E(\mathrm{eV})$ is the photoelectron energy. For isotropic scattering, the scattering angle is $\cos \theta=1-2 m$. The photons are assumed to be normally incident on the surface and the photoemitted electrons are isotropically distributed around the incident axis.

For inelastic scattering, the CSDA (Ref. 14) approximates the energy lost by an electron per unit distance traveled and is the stopping power (SP) for a material. Using the expression for photoelectron energy above, we estimate the photoemitted (which may or may not be expelled) electrons to have an energy of $\sim 15 \mathrm{eV}$ for $20 \mathrm{eV}$ incident photons. For $15 \mathrm{eV}$ electrons in $\mathrm{Si}_{3} \mathrm{~N}_{4}$, the $\mathrm{SP}$ is $0.064 \mathrm{eV} / \AA{ }^{14}$ The trajectory calculation ends when the electron energy drops below $8 \mathrm{eV}$ or when it escapes the dielectric. The $8 \mathrm{eV}$ lower limit is the approximate sum of the band-gap energy and the energy needed to expel the electron. Thus, for an SP of $0.064 \mathrm{eV} / \AA$, a $15 \mathrm{eV}$ electron can no longer be expelled after traveling a distance of $\sim 100 \AA$.

The other randomly generated parameters are: (1) The surface location of the incident photon on the dielectric, (2) the distance traveled by the photons before they interact with an atom, and (3) the distance and direction traveled by the electrons after each scattering event. The $20 \mathrm{eV}$ photons are assumed normally incident on a $\mathrm{Si}_{3} \mathrm{~N}_{4}$ dielectric of $3000 \AA$ thickness. Results show that a typical penetration depth of 20 $\mathrm{eV}$ photons is of the order of 80-100 A. For these conditions, the simulation predicts that $\sim 2.7 \%$ of the photoemitted electrons will be expelled from the dielectric.

Next, the maximum number of electrons that can be collected by an external circuit from those emitted from the dielectric was computed. This must be done separately because the simulation does not explicitly take into account the charge buildup on the surface of the dielectric due to the emission of photoelectrons.

In the experimental results shown in Fig. 1, the expelled electrons are collected by a plate located $3 \mathrm{~cm}$ above and to AlP license or copyright, see http://apl.aip.org/apl/copyright.jsp 
parallel to the dielectric and biased to $+48 \mathrm{~V}{ }^{3}$ The plate has a circular aperture to allow the VUV beam to reach the dielectric. By plotting the instantaneous value of the collected current versus substrate voltage, which can be obtained point by point from Fig. 1, it was observed that the collected (photoemission) current varies as $\Delta V^{3 / 2}$, where $\Delta V$ is the voltage between the plate and the substrate, i.e., the difference between the plate bias voltage and the substrate voltage. This dependence indicates that the current is space-charge limited and obeys the Child-Langmuir law. ${ }^{15}$ As the dielectric charges up, $\Delta V$ decreases with time, resulting in a decrease of the photoemission current shown in Fig. 1.

To compute the surface-charge density, we calculate the total number of electrons that have escaped from the dielectric and which traverse the space charge to be collected by the plate. It is assumed that an equivalent number of holes are left behind in the dielectric, thus forming a positive surface charge. Figure 2 shows that nearly all of the photoemitted electrons are produced inside of the area of the photon beam, thus we assume the charge is uniformly distributed over this area. We now calculate the surface charge density by using the following expression:

$$
\rho_{s}=\int_{0}^{t_{0}}\left[\eta \Gamma_{p} q_{e} / A\right] d t
$$

where $\rho_{s}$ is the charge density at time $t_{0} . \eta$ is the fraction of emitted electrons that are collected by the plate, and is proportional to the photoemission current and thus varies as $\Delta V^{3 / 2}$. The maximum value of $\eta$ occurred at $t=0$ when $\Delta V$ $=+48 \mathrm{~V}$ was 0.0267. In Eq. (5) $\Gamma_{p}$ is the photon flux $\left(5 \times 10^{10}\right.$ photons/s $), \quad q_{e}$ is the electronic charge, and $A\left(1.05 \mathrm{~cm}^{2}\right)$ is the photon-beam area. To find the total photoemitted charge, $t_{0}$ was set to $28 \mathrm{~s}$ and Eq. (5) yields a surface charge of $1.12 \mu \mathrm{C} / \mathrm{m}^{2}$. For this charge density, the Kelvin probe used in this experiment indicates a surface potential of $3.2 \mathrm{~V}$.

To compare the simulation with experiment, a silicon wafer coated with $3000 \AA$ of silicon nitride was exposed to $20 \mathrm{eV}$ photons. The wafer was exposed for $28 \mathrm{~s}$, which is approximately the time duration of the photoemission current shown in Fig. 1. The surface potential on the dielectric was measured with the Kelvin probe.,

The photon flux in the VUV beam is roughly Gaussian in shape. The background potential on the dielectric ranged from $0-1 \mathrm{~V}$. At the center of the beam, the surface potential was $2.65 \mathrm{~V}$, which is comparable to the potential obtained from the Monte Carlo simulation.

The surface potential computed from the simulation is well within a factor of 2 of the experimental measurements. Statistical information about the emitted electron percentage, photon penetration depth, and electron transport was obtained from the Monte Carlo simulation. The simulation can calculate the photoemission-induced charge for a range of dielectrics and photon energies so that it is possible to compute the photoemission charge produced by the exposure of a dielectric-coated wafer to VUV photons produced by plasma exposure during semiconductor processing.

This work was supported by the National Science Foundation under Grant No. DMR-0306582. The SRC is funded by a NSF grant (No. DMR-0084402). The authors would like to thank D. C. Joy, R. Hafner, R. D. Bathke, and R. W. Hansen for their assistance.

${ }^{1}$ C. T. Gabriel and J. P. McVittie, Solid State Technol. 35, 81 (1992).

${ }^{2}$ C. Cismaru and J. L. Shohet, Appl. Phys. Lett. 76, 2191 (2000).

${ }^{3}$ J. L. Lauer, J. L. Shohet, C. Cismaru, R. W. Hansen, M. Y. Foo, and T. J. Henn, J. Appl. Phys. 91, 1242 (2002).

${ }^{4}$ J. L. Lauer, J. L. Shohet, and R. W. Hansen, J. Vac. Sci. Technol. A 21, 1253 (2003).

${ }^{5}$ J. L. Lauer, J. L. Shohet, R. W. Hansen, R. D. Bathke, B. Grierson, G. Upadhyaya, K. Kukkady, and J. M. Kalwitz, Proceedings of the International Conference on Plasma and Process-Induced Damage, Austin, TX (2004), p. 307.

${ }^{6}$ F. James, Rep. Prog. Phys. 43, 73 (1980).

${ }^{7}$ S. M. Seltzer, Monte Carlo Transport of Electrons and Photons (Plenum, New York, 1988).

${ }^{8}$ C. T. Chantler, J. Phys. Chem. Ref. Data 24, 71 (1995).

${ }^{9}$ T. S. Rao-Sahib and D. B. Wittry, J. Appl. Phys. 45, 5060 (1974).

${ }^{10}$ C. T. Chantler, K. Olsen, R. A. Dragoset, A. R. Kishore, S. A. Kotochigova, and D. S. Zucker, X-Ray Form Factor, Attenuation and Scattering Tables (version 2.0). [Online] Available at 〈http://physics.nist.gov/ ffast>, NIST, Gaithersburg, MD. Originally published as C. T. Chantler, J. Phys. Chem. Ref. Data 29, 597 (2000); 24, 71 (1995).

${ }^{11}$ Z. Czyzewski, D. MacCalium, A. Romig, and D. C. Joy, J. Appl. Phys. 68, 3066 (1990).

${ }^{12}$ R. Browning, T. Eimori, E. P. Traut, B. Chui, and R. F. W. Pease, J. Vac. Sci. Technol. B 9, 3578 (1991).

${ }^{13}$ F. Arqueros and G. D. Montesinos, Am. J. Phys. 71, 38 (2003).

${ }^{14}$ J. C. Ashley and V. E. Anderson, J. Electron Spectrosc. Relat. Phenom. 24, 127 (1981).

${ }^{15}$ J. Millman, Vacuum-Tube and Semiconductor Electronics (McGraw-Hill, New York, 1958). 